\title{
ON BRITTON'S THEOREM A
}

\author{
CHARLES F. MILLER III ${ }^{1}$
}

In [1] Britton gives a "group-theoretic" proof of the unsolvability of the word problem for finitely presented groups. His proof uses a very powerful result [1, Lemma 4 (the principal lemma)] on presentations which has become known as Britton's Lemma and has since been used by several other authors. In the appendix to [1] Britton states a generalization of this lemma as Theorem A. Britton briefly indicates how Theorem A can be deduced from the previous lemma and how it may be further generalized. We give a direct proof of Theorem A in its most general form which specializes immediately to Britton's Lemma. Our proof appeals more directly to the theory of free products with amalgamation.

By $E=(S ; D)$ we mean that the group $E$ is presented by generators $S$ and relations $D$. By $G=(A * B ; H=K, \phi)$ we mean that $G$ is the free product of groups $A$ and $B$ amalgamating their subgroups $H$ and $K$ via the isomorphism $\phi$ ( $\phi$ will be omitted where the map is obvious from the context). The notation $w \equiv v$ will mean that $w$ and $v$ are identical as words, while $w=v$ refers to equality of words as elements of some appropriate group.

The following lemma is an immediate consequence of the normal form for free products with amalgamation:

Lemma 1. Suppose $G=\left(A_{1} * A_{2} ; H_{1}=H_{2}, \phi\right)$ and let $w \equiv h g_{1} \cdots g_{n}$ $(n \geqq 1)$, where $h \in H_{1}=H_{2}$ and $g_{i} \in A_{v(i)}$, where $v(i) \neq v(i+1)$, so that the $g_{i}$ lie in alternating factors. Then if $w=1$ in $G$ there is some $i$ such that $g_{i} \in H_{1}=H_{2}$.

$P$ will denote a set of letters indexed by a set $V$. We write $p(v)$ rather than $p_{v}$ to avoid several levels of subscripting. Let $E=(S ; D)$ be any presentation and assume $P$ and $S$ are disjoint. A presentation $E^{*}$ is said to have stable letters $P$ and corresponding basis $E$ if it has the form

$$
E^{*}=\left(S, P ; D, p\left(y_{i}\right)^{-1} A_{i} p\left(z_{i}\right)=B_{i}(i \in I)\right),
$$

where $y_{i}, z_{i} \in V$ and $A_{i}$ and $B_{i}$ are words over $S .{ }^{2}$

Received by the editors June 2, 1967.

1 This work was partially supported by National Science Foundation contract NSF-GP 6132.

2 The concept of stable letters is motivated by a similarity to inner automorphisms "and by an analogy to internal states of a Turing machine. See [3, Chapter 12], in this connection. 
We write $p(y) \sim p(z)$ if $p(y)=p(z)$ in the free group obtained from $E^{*}$ by setting all the letters of $S$ equal to 1 . Let $K(v)$ $=\left\{i \in I: p\left(y_{i}\right) \sim p(v)\right\}$ denote the induced equivalence classes in $I$. $A(v)$ denotes the subgroup of $E$ generated by the $A_{i}$ such that $i \in K(v)$, and similarly for $B(v)$.

$E^{*}$ as above satisfies the generalized isomorphism condition $(G I C)$ if for each $K(v)$ the map $A_{i} \rightarrow B_{i}(i \in K(v))$ defines an isomorphism between $A(v)$ and $B(v)$.

Let $T, U$ be words over $S$ and let $y, z \in V$. We say $T p(y)$ produces $p(z) U$ if the $T p(y)$ can be transformed into $p(z) U$ by a sequence of operations of the form

$$
X A_{i} p\left(z_{i}\right) Y \rightarrow X p\left(y_{i}\right) B_{i} Y \quad \text { or } X A_{i}^{-1} p\left(y_{i}\right) Y \rightarrow X p\left(z_{i}\right) B_{i}^{-1} Y,
$$

where $X, Y$ are words over $S$.

It can be shown that if $T p(y)$ produces $p(z) U$, then $T$ is a word in $A(y)$, say $T \equiv w\left(A_{i}\right)$, and $U$ the corresponding word $w\left(B_{i}\right)$ in $B(y)$. Moreover $T p(y)=p(z) U$ in $E^{*}$.

A word $W$ involves the letter $p$ if either $p$ or $p^{-1}$ is a subword of $W$. (In particular, the word $p p^{-1}$ involves $p$.)

Theorem A. Suppose $E^{*}$ as above satisfies the GIC. Then

(I) $E$ is embedded in $E^{*}$ and

(II) if $W=1$ in $E^{*}$ where $W$ involves at least one letter $p \in P$, then $W$ contains a subword (1) $p(y)^{-1} C p(z)$ or (2) $p(y) C p(z)^{-1}$ where $C$ is a word over $S$. In case (1), $C$ is equal in $E$ to a word $w\left(A_{i}\right) \in A(y)$ and $w\left(A_{i}\right) p(z)$ produces $p(y) w\left(B_{i}\right)$. In case (2), $C$ is equal in $E$ to a word $w\left(B_{i}\right) \in B(y)$ and $w\left(A_{i}\right) p(z)$ produces $p(y) w\left(B_{i}\right)$.

Proof. Let $[a(v)(v \in V)]$ denote the free group generated by the $a(v)$. Generally, $\langle Q\rangle$ denotes the subgroup generated by the set $Q$. Define the (ordinary) free products:

$$
F=[a(v)(v \in V)] * E, \quad G=[b(v)(v \in V)] * E .
$$

Consider the subgroups $M_{v}=\left\langle a\left(y_{i}\right)^{-1} A_{i} a\left(z_{i}\right)(i \in K(v))\right\rangle$ of $F$ and $N_{v}=\left\langle b\left(y_{i}\right) B_{i} b\left(z_{i}\right)^{-1}(i \in K(v))\right\rangle$ of $G$.

Then $M_{v}=M_{t}$ if and only if $K(v)=K(t)$. Also $\left\langle M_{v}, M_{t}\right\rangle \cong M_{v} * M_{t}$ if $K(v) \neq K(t)$ and $\left\langle M_{v}, E\right\rangle \cong M_{v} * E$ for each $v$ as subgroups of $F$. Similar considerations apply to the $N_{v}$ in $G$.

By the GIC it follows that $M_{\bullet} \cong N$ by the obvious map. Now we put

$$
\begin{aligned}
& Y=\left\langle a\left(y_{i}\right)^{-1} A_{i} a\left(z_{i}\right)(i \in I)\right\rangle * E \subset F, \\
& Z=\left\langle b\left(y_{i}\right) B_{i} b\left(z_{i}\right)^{-1}(i \in I)\right\rangle * E \subset G .
\end{aligned}
$$


Hence $Y \cong Z$ under the map $E \rightarrow E$ and $M_{v} \rightarrow N_{v}$, i.e., $a\left(y_{i}\right)^{-1} A_{i} a\left(z_{i}\right)$ $\rightarrow b\left(y_{i}\right) B_{i} b\left(z_{i}\right)^{-1}$ for each $i$. Now setting

$\bar{E}=\left(S, a(v), b(v)(v \in V) ; \quad D, a\left(y_{i}\right)^{-1} A_{i} a\left(z_{i}\right)=b\left(y_{i}\right) B_{i} b\left(z_{i}\right)^{-1}(i \in I)\right)$,

we see immediately that $\bar{E} \cong(F * G ; Y=Z)$. The map $S \rightarrow S, p(v)$ $\rightarrow a(v) b(v)$ embeds $E^{*}$ in $\bar{E}$. To see this, observe that the relations of $\bar{E}$ are exactly the relations of $E^{*}$ under this map, so it is certainly a homomorphism. Moreover, if a word $W(S, p(v)) \rightarrow W(S, a(v) b(v))=1$ in $\bar{E}, W$ must also equal 1 in the group $T$ obtained from $\bar{E}$ by setting $b(v)=1$. But clearly $T \cong E^{*}$ via the map $S \rightarrow S, a(v) \rightarrow p(v)$. Since the composite of all these maps is the identity on $E^{*}, W(S, p(v))=1$ in $E^{*}$. Hence $E$ is embedded in $E^{*}$ and $\bar{E}$ by $S \rightarrow S$ for $E \subset Y=Z$.

Now to prove the theorem we may assume $W$ is freely reduced in $E^{*}$ and

$$
W \equiv S_{1} p\left(v_{1}\right)^{\alpha_{1}} S_{2} p\left(v_{2}\right)^{\alpha_{2}} \cdots S_{n} p\left(v_{n}\right)^{\alpha_{n}} S_{n+1},
$$

where $n \geqq 1$ and all $\alpha_{i} \neq 0$ and $S_{i} \in E$. Since $W=1$ in $E^{*}$ we must have for its embedded image in $\bar{E}$

$$
W \equiv S_{1}\left(a\left(v_{1}\right) b\left(v_{1}\right)\right)^{\alpha_{1}} S_{2} \cdots S_{n}\left(a\left(v_{n}\right) b\left(v_{n}\right)\right)^{\alpha_{n}} S_{n+1}=1 \quad \text { in } \bar{E} .
$$

Notice that $a(v) \in F \backslash Y, \quad b(v) \in G \backslash Z$ and $S_{i} \in Y=Z$ since $\bar{E}$ $\cong(F * G ; Y=Z)$. By hypothesis $n \geqq 1$. From Lemma 1 we conclude that for some $i$ one of the following holds:

(1) $\alpha_{i}<0$ and $\alpha_{i+1}>0$ and $a\left(v_{i}\right)^{-1} S_{i+1} a\left(v_{i+1}\right) \in Y$.

(2) $\alpha_{i}>0$ and $\alpha_{i+1}<0$ and $b\left(v_{i}\right) S_{i+1} b\left(v_{i+1}\right)^{-1} \in Z$.

(3) $\alpha_{i}<0$ and $\alpha_{i+1} \leqq 0$ and $a\left(v_{i}\right)^{-1} S_{i+1} \in Y$.

(4) $\alpha_{i}>0$ and $\alpha_{i+1} \geqq 0$ and $b\left(v_{i}\right) S_{i+1} \in Z$.

But cases (3) and (4) are clearly impossible. So consider case (1). It follows from notation and free products that $a\left(v_{i}\right)^{-1} S_{i+1} a\left(v_{i+1}\right) \in M_{v}$ for some $v$, since $Y \cong E * R$ where $R$ is the free product of the distinct $M_{v}$ and such a word must have free product normal form length 1 (cancelling $a(v)$ pairs must come from generators of the same $M_{v}$ by our notation). Hence $S_{i+1}=w\left(A_{l}\right)$ in $E$ for $w\left(A_{l}\right) \in A(v)$. Indeed, we have

$$
a\left(v_{i}\right)^{-1} S_{i+1} a\left(v_{i+1}\right)=\prod_{j=1}^{m} a\left(t_{j}\right)^{-1} A_{l_{j}}^{e_{j}} a\left(t_{j+1}\right)
$$

and

$$
w\left(A_{l}\right) \equiv \prod_{j=1}^{m} A_{l_{j}}^{\iota_{j}}, \quad \text { where } \quad \epsilon_{j}= \pm 1
$$

and $a\left(v_{i}\right)=a\left(t_{1}\right)$ and $a\left(t_{m+1}\right)=a\left(v_{i+1}\right)$ and where the $a\left(t_{j}\right)^{-1} A_{l_{j}}^{c_{j}} a\left(t_{j+1}\right)$ 
are generators of $M_{v}$ or their inverses. Hence $W$ contains a subword equal to

$$
b\left(v_{i}\right)^{-1} a\left(v_{i}\right)^{-1} w\left(A_{l}\right) a\left(v_{i+1}\right) b\left(v_{i+1}\right),
$$

which as a word of $E^{*}$ has the form $p\left(v_{i}\right)^{-1} w\left(A_{l}\right) p\left(v_{i+1}\right)$. But in $\bar{E}$ we have

$$
\begin{aligned}
a\left(v_{i}\right)^{-1} S_{i+1} a\left(v_{i+1}\right) & =a\left(v_{i}\right)^{-1} w\left(A_{l}\right) a\left(v_{i+1}\right) \\
& =b\left(v_{i}\right) w\left(B_{l}\right) b\left(v_{i+1}\right)^{-1},
\end{aligned}
$$

where $w\left(A_{l}\right) \in A(v)$ and $w\left(B_{l}\right) \in B(v)$. Moreover, $p\left(v_{i}\right)^{-1} w\left(A_{l}\right) p\left(v_{i+1}\right)$ $=w\left(B_{i}\right)$ and $w\left(A_{l}\right) p\left(v_{i+1}\right)$ produces $p\left(v_{i}\right) w\left(B_{l}\right)$ by use of the relations corresponding to the sequence of $a\left(t_{j}\right)$ 's. This completes the proof in the situation arising in case (1) of the theorem.

Similarly, case (2) can be handled by the dual proof.

Corollary 1. Let $E^{*}$ and $E$ be as in Theorem A. Let $n$ be a positive integer. Then $E^{*}$ has elements of order $n$ if and only if $E$ has elements of $\operatorname{order} n$.

Proof. The claim is true for $\bar{E}$ (and hence $E^{*}$ ) by properties of free products with amalgamation, see [2].\|

A group $G=E_{n} \geqq E_{n-1} \geqq \cdots \geqq E_{0}=E$ is called a Britton tower over $E$ if each $E_{i} \geqq E_{i-1}$ satisfies the conditions for $E^{*} \geqq E$ of Theorem A.

Corollary 2. Let $G$ be a Britton tower over $E$. Let $n$ be a positive integer. Then $G$ has elements of order $n$ if and only if $E$ has elements of order $n$.

Proof. By induction and Corollary 1.\|

In the special case

$$
E^{*}=\left(S, P ; D, p\left(y_{i}\right)^{-1} A_{i} p\left(y_{i}\right)=B_{i}(i \in I)\right),
$$

we have $p(v) \sim p(w)$ if and only if $p(v)=p(w)$. Then the GIC becomes the isomorphism condition of Britton (see [1] or [3, Chapter 12]). Also $T p(v)$ produces $p(w) U$ implies $p(v)=p(w)$. Hence Theorem A just becomes Britton's Lemma. A consequence of $E$ being embedded in $E^{*}$ is a well-known theorem of Higman, Neumann and Neumann (see [3, Chapter 11]).

\section{REFERENCES}

1. J. L. Britton, The word problem, Ann. of Math. 77 (1963), 16-32.

2. W. Magnus, A. Karrass and D. Solitar, Combinatorial group theory, Wiley, New York, 1966.

3. J. Rotman, The theory of groups, Allyn and Bacon, Boston, Mass., 1965; Chapters 11 and 12 .

UNiversity OF ILLINOIS 